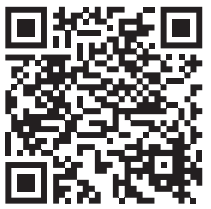

Palabras clave: Realidad virtual, validez de constructo, simulación, cirugía de cataratas.

Keywords: Virtual reality, construct validity, simulation, cataract surgery.

\footnotetext{
* Consultor y

Voluntario Facultativo, Flying Eye Hospital, Orbis International. Oftalmólogo y Entrenador Quirúrgico Pontificia Universidad Católica de Chile/

Clínica Oftalmológica, Luis Pasteur.

¥ Jefe de Oftalmología, Flying Eye Hospital, Orbis International.

$\S$ Vicepresidente de Servicios Clínicos, Orbis International.
}

Recibido: 14/05/2020

Aceptado: 20/07/2020

doi: 10.35366/95231

\title{
Validez de constructo del uso de realidad virtual en la cirugía de cataratas: revisión sistemática
}

\author{
Construct validity of the use of virtual reality in \\ cataract surgery: systematic review \\ Andreas Di-Luciano, ${ }^{*}$ María Montero, ${ }^{\ddagger}$ Hunter Chewerk ${ }^{\S}$
}

\section{RESUMEN}

Introducción: El uso de la realidad virtual (RV) en el entrenamiento quirúrgico ha ido ganando cada vez más popularidad. El uso de RV en mallas curriculares es un hecho y la necesidad de comprobar el impacto real en el entrenamiento también. Es por ello que el objetivo de este manuscrito es realizar una revisión sistemática de la literatura sobre la validez de constructo en el uso de RV en cirugía de cataratas. Material y métodos: Revisión sistemática en PubMed y la Biblioteca Cochrane para estudios publicados del 1 de enero de 2008 al 1 de enero de 2018. Las palabras clave de búsqueda: simulation in ophthalmology, virtual reality, construct validity y cataracts surgery. Los criterios de inclusión para esta búsqueda fueron los siguientes: 1 ) artículos publicados en los últimos 10 años, 2) relacionados con la simulación de realidad virtual en cirugía de cataratas y 3 ) artículos que evalúan la validez constructiva. También incluimos artículos sobre análisis de costos y herramientas de aprendizaje que podrían asociarse con una capacitación de simulación. Después de la búsqueda, encontramos 52 artículos de los cuales utilizamos 15 para el análisis sistemático y 21 para la discusión y desarrollo del tema. Resultados: Los módulos que han mostrado una validez de constructo son antitemblor y fórceps, capsulorrexis, maniobras de hidratación, cracking y chopping $(\mathrm{p}<0.05)$. El uso de RV en la cirugía de cataratas ha mostrado buenos resultados en pacientes reales a través de una tasa más baja de ruptura de la cápsula posterior ( $\mathrm{p}=$ 0.032), pérdida de vítreo, menos tiempo de faco $(p=0.02)$ y tiempo de operación $(\mathrm{p}=0.001)$. También se informa una mayor confianza de los cirujanos en los pasos de la cirugía. Evaluar el rendimiento con RV es de suma importancia ya que determinará en parte la preparación de las nuevas generaciones de cirujanos y los resultados quirúrgicos futuros de los pacientes. La evaluación estructurada objetiva de la habilidad quirúrgica de cataratas (OSACSS) y la evaluación estructurada objetiva de las habilidades quirúrgicas técnicas (OSATS) se han utilizado para evaluar el rendimiento quirúrgico en los residentes. Conclusiones: El uso de la realidad virtual en cirugías de cataratas es una herramienta que ya ha demostrado su validez de constructo. Consideramos que es una alternativa importante (complemento) al entrenamiento basado en ojos artificiales o animales. Se necesitan estudios a más largo plazo con diseños más estandarizados para evaluar su impacto real y rentabilidad.

\section{ABSTRACT}

Introduction: The use of virtual reality (VR) in surgical training has been gaining popularity. The use of $V R$ is used today as part of surgical training programs in many centers. For this reason, the need arises to evaluate the real impact of VR on training in cataract surgery. The objective of this manuscript, is to carry out a systematic review of the literature on construct validity in the use of VR in cataract surgery. Material and methods: Systematic review was done using PubMed and the Cochrane Library for studies published from January 1, 2008 to January 1, 2018. Search keywords were: simulation in ophthalmology, virtual reality, construct validity and cataract surgery. The inclusion criteria were the following: (1) articles published in the last 10 years; (2) related to virtual reality simulation in cataract surgery and (3) articles that evaluate construct validity. We also include articles on cost analysis and learning tools that could be associated with simulation training. After the search, we found 52 articles of which we used 15 for the systematic review and 21 for the discussion. Results: The modules that have shown constructive validity are anti-tremor and forceps, capsulorexis, hydration maneuvers, cracks and chopping $(p<0.05)$. The use of VR in cataract surgery has shown good results in real patients through a lower posterior capsule rupture rate $(p=0.032)$, vitreous loss, less phaco time $(p=0.02)$ and time of operation $(p=0.001)$. Surgeons' increased confidence in the steps of surgery are also reported. Assessing performance with VR is of utmost importance as it will partly determine the readiness of new generations of surgeons and the future surgical outcomes of patients. Objective Structured Assessment of Cataract Surgical Ability (OSACSS) and Objective Structured Assessment of Technical Surgical Skills (OSATS) have been used to assess surgical performance in residents. Conclusions: The use of virtual reality in cataract surgeries is a tool that has already demonstrated its construct validity. We consider it an important alternative (complement) to training based on artificial or animal eyes. Longer-term studies with more standardized designs are needed to assess their real impact and cost effectiveness. 


\section{INTRODUCCIÓN}

$E^{\prime}$ concepto de simulación se remonta al año 1929 cuando Edward Link creó un sistema de simulación para evitar accidentes aéreos. ${ }^{1}$ La historia de la simulación en oftalmología comienza en 1992 con la creación de un simulador para inyección de anestesia retrobulbar llamado ORIS. ${ }^{2}$ Un año después, Hunter y colaboradores crearon un robot microquirúrgico teleoperado para cirugía ocular. ${ }^{3}$

El uso de la realidad virtual (RV) ya ha demostrado ser exitoso en otras ramas de la medicina como Urología, ${ }^{4}$ Cirugía General, ${ }^{5}$ Ginecología, ${ }^{6}$ Otorrinolaringología, 7 Anestesiología ${ }^{8}$ y más.

El aumento en la popularidad del uso de RV en el entrenamiento quirúrgico en pregrado es un hecho. Esto se refleja en el aumento de la implementación de realidad virtual en los programas de residencia en oftalmología en todo el mundo; sin embargo, la evidencia que existe hoy en la literatura no es extensa. En la última década, se han realizado estudios que buscan demostrar la validez constructiva de los simuladores en la realidad virtual. La gran mayoría de los estudios se han realizado con el Eyesi (VR Magic, Gmbh, Mannheim, Alemania). ${ }^{9-19}$ Se ha demostrado la validez constructiva de algunos módulos para este simulador, tales como: capsulorrexis, ${ }^{10-12}$ antitemblor y fórceps, ${ }^{9}$ maniobras de hidratación, cracking y chopping. ${ }^{13}$

El análisis de costos de implementación de un programa de simulación con RV, así como la evaluación de su impacto en el proceso de aprendizaje son factores a considerar. ${ }^{20}$

Por lo tanto, realizamos una revisión sistemática en la literatura para evaluar la validez de constructo del uso de RV en oftalmología en cirugía de cataratas. También evaluamos las herramientas utilizadas para evaluar y aprender el uso de esta tecnología y el análisis de costos de su implementación.

\section{MATERIAL Y MÉTODOS}

El propósito de esta revisión es evaluar la validez de constructo en el uso de realidad virtual en cirugía de cataratas. La validez de constructo se define como un instrumento para medir si las inferencias hechas sobre la base de observaciones y/o mediciones mide el constructo que se busca. La validez de constructo generalmente se verifica comparando la prueba con otras que miden cualidades similares para ver cuán altamente correlacionadas están las dos medidas. En este caso, son los módulos de cirugía de cataratas los cuales a través de su software de evaluación pueden obtener medidas numéricas comparables a través del desempeño del practicante en dichas tareas.

\section{Estrategia de búsqueda y selección de estudios}

Se realizó una búsqueda sistemática en las bases de datos de PubMed/Medline, PubMed and the Cochrane Library, de documentos publicados del 1 de enero de 2008 al 1 de enero de 2018, utilizando las siguientes palabras claves: simulation in ophthalmology, virtual reality, construct validity y cataracts surgery. Adicionalmente, se revisó la lista de referencias de los estudios identificados y de las revisiones relevantes en el tema buscando otros posibles estudios que pudieran ser incluidos.

Se incluyeron estudios de tipo ensayo clínico aleatorizado (ECA), estudios de cohortes, y series de casos, que cumplieran los siguientes criterios de inclusión: 1) artículos publicados en los últimos 10 años, 2) relacionados con la simulación de realidad virtual en cirugía de cataratas y 3) artículos que evalúan la validez constructiva. También incluimos artículos sobre análisis de costos y herramientas de aprendizaje que podrían asociarse con una capacitación de simulación. Se excluyeron estudios duplicados y con datos incompletos. Referente al idioma, se utilizaron estudios en español e inglés. La evaluación de la calidad de los estudios se realizó según la clasificación de los niveles de evidencia del Centre for Evidence-Based Medicine, Oxford (OCEBM). La selección de los estudios fue realizada de forma independiente por dos investigadores (AD y MM), las diferencias fueron resueltas por un tercer investigador $(\mathrm{HC})$.

\section{Extracción de datos y análisis}

Los estudios encontrados fueron exportados al software EndNote X9 para eliminación de artículos duplicados y subsecuentemente importando a Number v6.1 6369 para su tabulación. Los datos extraídos de los estudios fueron: autor principal, año de publicación, metodología, tipo de simulador utilizado, número de pacientes, resultados midiendo la validez de constructo y sus conclusiones.

Los datos extraídos fueron revisados por dos investigadores de forma independiente (AD, $M M)$, al existir alguna observación sobre uno de 
los estudios fue evaluado una vez más por otro investigador $(\mathrm{HC})$. La revisión fue evaluada una vez más por expertos en el tema (AG) antes de crear el manuscrito final.

Los datos fueron agrupados en tablas descriptivas utilizando el software Numbers v6.1 6369 (Apple Inc). Dada la heterogeneidad de los estudios, no se realizó metaanálisis.

\section{RESULTADOS}

Con la estrategia de búsqueda se identificaron 52 estudios, de los cuales 16 fueron eliminados por duplicidad. Durante la revisión del título y resumen se encontraron 36 estudios potenciales. Finalmente, con la revisión de texto completo, fueron incluidos 15 artículos para la revisión (Figura 1). La mayoría de los artículos correspondieron a estudios prospectivos $(n=10)$ en donde se incluían estudios intervencionistas, cohortes y ECA. El nivel de evidencia fue en su mayoría nivel de evidencia 2 b y 3 b según la clasificación de la OCEBM. Dentro de las características de los estudios encontrados, el $93.33 \%$ fueron realizados utilizando el simulador de cirugía de cataratas Eyesi (VR Magic, Gmbh, Mannheim, Alemania), la

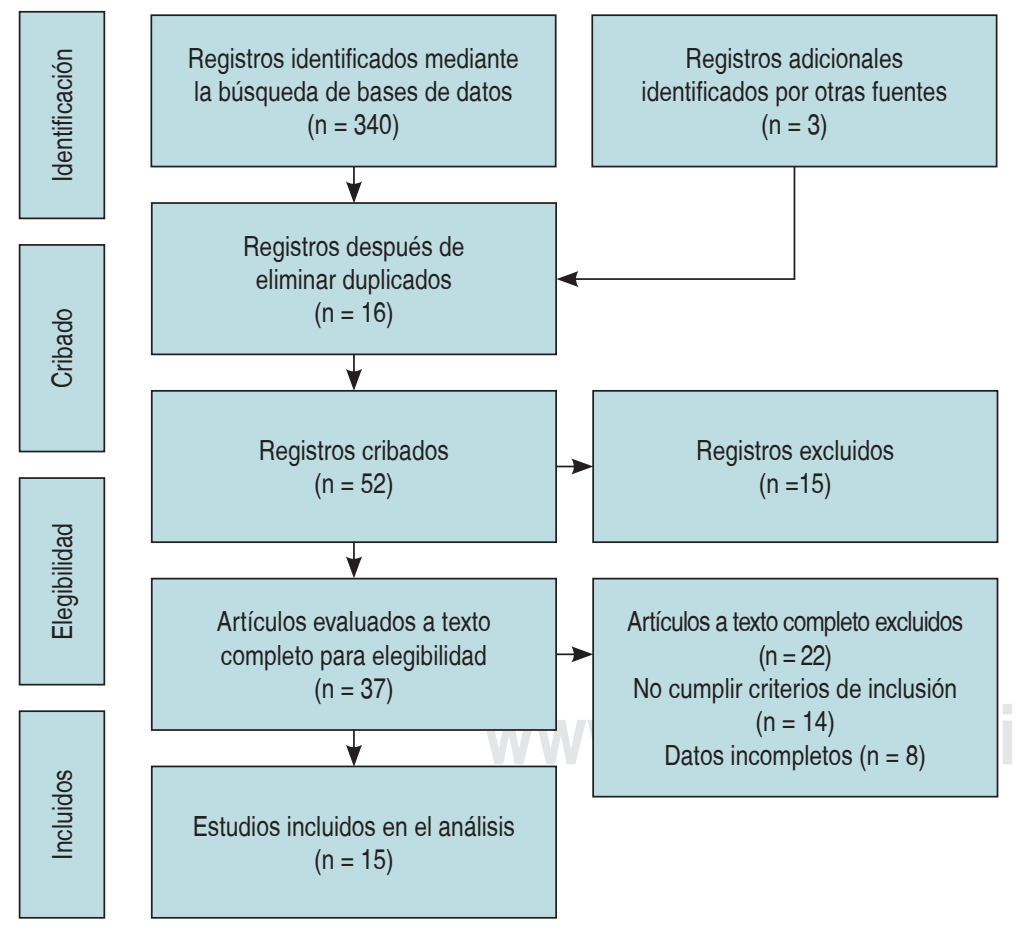

Figura 1: Diagrama de Flujo para la inclusión de los estudios en la revisión sistemática según las recomendaciones PRISMA. población de estudios incluyendo los casos y controles superó 20 participantes en el 100\% de los estudios y la validez de constructo fue evaluada en un $60 \%$ de los artículos seleccionados. Dentro de los estudios, los módulos que demostraron tener una validez de constructo fueron: antitemblor y fórceps, capsulorrexis, maniobras de hidratación, cracking y chopping $(\mathrm{p}<0.05)$. En relación con los resultados, al comparar el uso de RV y su impacto en cirugía de cataratas en pacientes reales, encontramos una tasa más baja de ruptura de la cápsula posterior $(p=0.032)$, pérdida de vítreo, menos tiempo de faco $(p=0.02)$ y tiempo de operación $(p=0.001)$. Las características de los estudios incluidos en el análisis se resumen en la Tabla 1.

\section{Evaluación de la validez de constructo en el uso de simulación con RV en cirugía de catarata con técnica de facoemulsificación}

En oftalmología, actualmente hay disponibles tres simuladores de cirugía de cataratas bajo técnica de facoemulsificación: Eyesi (VRmagic Holding AG, Mannheim, Alemania), MicroVisTouch (ImmersiveTouch, Chicago, IL) y PhacoVision (Melerit Medical, Linkoping, Suecia). El Eyesi consiste en un sistema de entrenamiento para la cataratas y retina, en cambio MicroVisTouch y PhacoVision se enfocan sólo en la catarata. Con respecto a los módulos Eyesi que han demostrado tener una validez de constructo, encontramos los siguientes estudios: Mahr y colaboradores encontraron que el simulador quirúrgico Eyesi en los módulos de manejo en el segmento anterior y antitemblor (Figura 2) tenían una validez de constructo significativa con un $\mathrm{p}<0.05$. $^{9}$

Se sabe que uno de los pasos más difíciles de aprender en la cirugía de cataratas es la capsulorrexis. Selvander y su equipo y Privett demostraron la validez de constructo de los módulos de capsulorrexis en Eyesi $(\mathrm{p}<0.05) .{ }^{10,11}$ Por otro lado, Feudner y su grupo de investigadores encontraron que el uso complementario del módulo de capsulorrexis (Figura 3) en Eyesi se correlacionó positivamente con la práctica de capsulorrexis en el Wet-lab $(p=0.001){ }^{12}$

Otro estudio realizado por Selvander en el año 2013 demostró la validez de constructo de otros módulos Eyesi como los de maniobras de hidratación, cracking (Figura 4) y chopping y navegación en el segmento anterior $(p<0.05) .{ }^{13}$

Con respecto a la extrapolación del uso de ciertos módulos Eyesi a cirugías con pacientes 


\begin{tabular}{|c|c|c|c|c|c|}
\hline $\begin{array}{l}\text { Autor } \\
\text { y año }\end{array}$ & $\begin{array}{l}\text { Diseño } \\
\text { metodológico }\end{array}$ & Simulador & Número participantes & Resultados & Conclusiones \\
\hline $\begin{array}{l}\text { Mahr et al } \\
2008^{9}\end{array}$ & $\begin{array}{l}\text { Prospectivo, } \\
\text { comparativo }\end{array}$ & $\begin{array}{l}\text { Eyesi } \\
\text { Catarata }\end{array}$ & $\begin{array}{l}12 \text { residentes y } 5 \text { cirujanos experimen- } \\
\text { tados }\end{array}$ & $\begin{array}{l}\text { Cirujanos experimentados con mejor } \\
\text { resultados en módulos de antitemblor }(\mathrm{p} \\
=0.02) \text { y módulos de fórceps }(\mathrm{p}=0.05)\end{array}$ & $\begin{array}{l}\text { Módulos de antitemblor y fórceps con } \\
\text { validez de constructo positiva }\end{array}$ \\
\hline $\begin{array}{l}\text { Feudner et } \\
\text { al } 2009^{12}\end{array}$ & $\begin{array}{l}\text { Prospectivo, randomiza- } \\
\text { do, enmascarado }\end{array}$ & $\begin{array}{l}\text { Eyesi } \\
\text { Catarata }\end{array}$ & $\begin{array}{l}31 \text { estudiantes de medicina y } 32 \text { resi- } \\
\text { dentes de oftalmología }\end{array}$ & $\begin{array}{l}\text { Mejoría en la realización de capsulo- } \\
\text { rrexis en Wetlab }(\mathrm{p}=0.001)\end{array}$ & $\begin{array}{l}\text { El uso de RV en módulos de capsulorrexis } \\
\text { mejora el desempeño en Wetlab }\end{array}$ \\
\hline $\begin{array}{l}\text { Privett } \\
\text { et al } \\
2010^{11}\end{array}$ & $\begin{array}{l}\text { Prospectivo, serie de } \\
\text { casos comparativos }\end{array}$ & $\begin{array}{l}\text { Eyesi } \\
\text { Catarata }\end{array}$ & $\begin{array}{l}\text { Grupo 1: estudiantes de medicina y } \\
\text { residentes de primer año }(\mathrm{n}=16) \\
\text { Grupo 2: cirujanos practicantes de } \\
\text { catarata }(\mathrm{n}=7)\end{array}$ & $\begin{array}{l}\text { Cirujanos practicantes de cataratas con } \\
\text { mejores resultados que estudiantes y } \\
\text { residentes en módulos de capsulorrexis } \\
(\mathrm{p}<0.05)\end{array}$ & $\begin{array}{l}\text { Validez de constructo positiva en } \\
\text { módulo de capsulorrexis }\end{array}$ \\
\hline $\begin{array}{l}\text { Belyea } \\
\text { et al } \\
2011^{14}\end{array}$ & $\begin{array}{l}\text { Prospectivo, serie de } \\
\text { casos comparativos }\end{array}$ & $\begin{array}{l}\text { Eyesi } \\
\text { Catarata }\end{array}$ & $\begin{array}{l}\text { Grupo A (RV) ( } \mathrm{n}=17 \text { residentes }) \\
\text { Grupo B (no RV) ( } \mathrm{n}=25 \\
\text { residentes) }\end{array}$ & $\begin{array}{l}\text { Grupo A, menos tiempo medio de faco } \\
(p=0.02) \text {, menos tiempo ajustado de } \\
\text { faco }(p=0.001) \text { y menos porcentaje } \\
\text { de poder de faco }(p=0.001)\end{array}$ & $\begin{array}{l}\text { Grupo A, mejores resultados que } \\
\text { Grupo B, en las variables de tiempo } \\
\text { medio, tiempo ajustado y porcentaje de } \\
\text { poder de faco }\end{array}$ \\
\hline $\begin{array}{l}\text { Lee et al } \\
2009^{28}\end{array}$ & $\begin{array}{l}\text { Estudio transversal } \\
\text { multicéntrico }\end{array}$ & $\begin{array}{l}\text { Eyesi } \\
\text { Catarata }\end{array}$ & $\begin{array}{l}\text { Cuatro estudiantes de medicina, } 4 \\
\text { aprendices de tecnología médica, } 36 \\
\text { residentes de oftalmología, } 3 \text { becarios y } \\
18 \text { oftalmólogos (Grupo A: aprendices } \\
\text { de TMO; Grupo B: residentes menores, } \\
\text { Grupo C: residentes y becarios mayores } \\
\text { y Grupo D: oftalmólogo del personal) }\end{array}$ & $\begin{array}{l}\text { Grupo D (oftalmólogo del personal) } \\
\text { mejores resultados en toda la tarea (p } \\
<0.05 \text { ) }\end{array}$ & $\begin{array}{l}\text { El nivel de entrenamiento de los } \\
\text { participantes es un predictor confiable } \\
\text { de su desempeño en los módulos de } \\
\text { antitemblor y fórceps }(\mathrm{p}<0.05)\end{array}$ \\
\hline $\begin{array}{l}\text { Selvander } \\
\text { et al } \\
2012^{10}\end{array}$ & $\begin{array}{l}\text { Prospectivo, serie de } \\
\text { casos comparativos }\end{array}$ & $\begin{array}{l}\text { Eyesi } \\
\text { Catarata }\end{array}$ & $\begin{array}{l}7 \text { cirujanos de cataratas y } 17 \text { estudian- } \\
\text { tes de medicina }\end{array}$ & $\begin{array}{l}\text { Mejora en faco, maniobras de hidrata- } \\
\text { ción, cracking, chopping y navegación } \\
\text { en cámara anterior }(\mathrm{p}<0.05)\end{array}$ & $\begin{array}{l}\text { Validez de constructo positiva en: faco, } \\
\text { maniobras de hidratación, cracking, chop- } \\
\text { ping y navegación en cámara anterior }\end{array}$ \\
\hline $\begin{array}{l}\text { Podbieslki } \\
\text { et al } \\
2012^{35}\end{array}$ & $\begin{array}{l}\text { Estudio de cohorte } \\
\text { prospectivo, desen- } \\
\text { mascarado e interven- } \\
\text { cionista }\end{array}$ & $\begin{array}{l}\text { Eyesi } \\
\text { Catarata }\end{array}$ & 18 residentes de oftalmología & $\begin{array}{l}\text { Para el módulo de destreza, no hubo } \\
\text { diferencias significativas }(\mathrm{p}>0.05)\end{array}$ & $\begin{array}{l}\text { Durante la cirugía intraocular simu- } \\
\text { lada, las herramientas quirúrgicas } \\
\text { activadas de manos y pies parecen } \\
\text { tener características de rendimiento } \\
\text { similares y son igualmente bien recibi- } \\
\text { das por los residentes }\end{array}$ \\
\hline $\begin{array}{l}\text { Pokroy } \\
\text { et al } \\
2012^{36}\end{array}$ & $\begin{array}{l}\text { Retrospectivo, com- } \\
\text { parativo }\end{array}$ & $\begin{array}{l}\text { Eyesi } \\
\text { Catarata }\end{array}$ & 20 residentes de oftalmología & $\begin{array}{l}\text { Tasas de desgarro capsular para los } \\
\text { grupos de simuladores y no simuladores } \\
\text { fueron } 8.8 \text { y } 10 \% \text { respectivamente para } \\
\text { los primeros } 25 \text { casos, y } 7.2 \text { y } 3.6 \% \text { ( }= \\
0.11 \text { ) respectivamente para los casos } 26 \\
\text { a } 50 \text {. El porcentaje de casos largos ( }>40 \\
\text { min) para los casos del } 10 \text { al } 50 \text { fue } 42.3 \\
\text { y } 32.4 \% \text { (p = } 0.005 \text { ) respectivamente }\end{array}$ & $\begin{array}{l}\text { El uso de RV acorta levemente la } \\
\text { curva de aprendizaje en los primeros } \\
50 \text { casos }\end{array}$ \\
\hline $\begin{array}{l}\text { McCannel } \\
\text { at al } \\
2013^{15}\end{array}$ & $\begin{array}{l}\text { Serie de casos inter- } \\
\text { vencionistas educati- } \\
\text { vos retrospectivos }\end{array}$ & $\begin{array}{l}\text { Eyesi } \\
\text { Catarata }\end{array}$ & $\begin{array}{l}1,037 \text { cirugías de cataratas } \\
\text { Cohorte basal }(n=434) \\
\text { Cohorte post intervención }(n=603)\end{array}$ & $\begin{array}{l}\text { Hubo } 15.7 \% \text { en la cohorte basal y } \\
5.0 \% \text { de CCC errantes }(\mathrm{p}<0.0001) \\
\text { en la cohorte post-intervención, una } \\
\text { reducción de } 3.2 \text { veces o } 68 \%\end{array}$ & $\begin{array}{l}\text { El entrenamiento de simulación } \\
\text { quirúrgica de realidad virtual con el } \\
\text { CITC en el Eyesi reduce la tasa de } \\
\text { capsulorrexis errante }\end{array}$ \\
\hline
\end{tabular}


Continua la Tabla 1: Agrupación de artículos.

\begin{tabular}{|c|c|c|c|c|c|}
\hline $\begin{array}{l}\text { Autor } \\
\text { y año }\end{array}$ & $\begin{array}{l}\text { Diseño } \\
\text { metodológico }\end{array}$ & Simulador & Número participantes & Resultados & Conclusiones \\
\hline $\begin{array}{l}\text { Thomsen } \\
\text { et al } \\
2015^{18}\end{array}$ & $\begin{array}{l}\text { Estudio intervencio- } \\
\text { nista prospectivo y } \\
\text { controlado }\end{array}$ & $\begin{array}{l}\text { Eyesi } \\
\text { Catarata }\end{array}$ & $\begin{array}{l}\text { Grupo A: principiantes }(n=26) \\
\text { Grupo B: cirujanos experimentados de } \\
\text { cataratas }(n=11) \\
\text { Grupo C: experimentados cirujanos de } \\
\text { realidad virtual }(n=5)\end{array}$ & $\begin{array}{l}\text { En } 7 \text { de } 13 \text { módulos, el grupo B tiene } \\
\text { mejores resultados que el Grupo A (p } \\
<0.05)\end{array}$ & $\begin{array}{l}\text { Se establece una prueba de rendimien- } \\
\text { to, que consta de siete módulos en el } \\
\text { simulador Eyesi Cataract }\end{array}$ \\
\hline $\begin{array}{l}\text { Sikder } \\
\text { et al } \\
2015^{25}\end{array}$ & $\begin{array}{l}\text { Prospectivo, rando- } \\
\text { mizado }\end{array}$ & $\begin{array}{l}\text { MicroVis } \\
\text { touch }\end{array}$ & $\begin{array}{l}8 \text { residentes de oftalmología en los } \\
\text { EUA y Arabia Saudita en la primera } \\
\text { ronda de pruebas y } 40 \text { residentes en } \\
\text { una segunda ronda de seguimiento }\end{array}$ & $\begin{array}{l}\text { Los puntajes promedio en todas las } \\
\text { métricas medidas demostraron una } \\
\text { mejora estadísticamente significativa } \\
\text { (excepto la circularidad, que tendió } \\
\text { a mejorar) después de la evaluación } \\
\text { inicial }\end{array}$ & No se valoró validez de constructo \\
\hline $\begin{array}{l}\text { Thomsen } \\
\text { et al } \\
2017^{18}\end{array}$ & $\begin{array}{l}\text { Ensayo clínico enmas- } \\
\text { carado multicéntrico }\end{array}$ & $\begin{array}{l}\text { Eyesi } \\
\text { Catarata }\end{array}$ & $\begin{array}{l}18 \text { cirujanos de catarata (diferentes } \\
\text { niveles) }\end{array}$ & $\begin{array}{l}\text { Los novatos y los cirujanos que } \\
\text { realizaron menos de } 75 \text { cirugías de } \\
\text { cataratas independientes mostraron } \\
\text { mejoras significativas en el quirófano } \\
\text { después del entrenamiento de realidad } \\
\text { virtual ( } \mathrm{p}=0.008 \text { y p }=0.018 \text { ). Con } \\
\text { respecto a los cirujanos de cataratas } \\
\text { experimentados, no se beneficiaron de } \\
\text { la capacitación en simulador }\end{array}$ & $\begin{array}{l}\text { Las habilidades quirúrgicas de cata- } \\
\text { ratas se pueden mejorar mediante un } \\
\text { entrenamiento basado en la competen- } \\
\text { cia en un simulador de realidad virtual. } \\
\text { Los principiantes y los cirujanos con } \\
\text { un nivel intermedio de experiencia } \\
\text { mostraron una mejora en la puntuación } \\
\text { de rendimiento OR }\end{array}$ \\
\hline $\begin{array}{l}\text { Starapoli } \\
\text { et al } \\
2018^{17}\end{array}$ & Retrospectivo & $\begin{array}{l}\text { Eyesi } \\
\text { Catarata }\end{array}$ & $\begin{array}{l}\text { Residentes novatos de postgrado año } \\
\text { 3. Grupo entrenado en simulador ( } n= \\
\text { 11). Grupo de comparación ingenuo } \\
\text { simulador }(n=11)\end{array}$ & $\begin{array}{l}\text { La tasa de complicaciones en el grupo } \\
\text { simulador }(p=0.037) \\
\text { Menos desgarro de la cápsula posterior } \\
\text { y prolapso vítreo }(p=0.032)\end{array}$ & $\begin{array}{l}\text { La adición del entrenamiento de } \\
\text { simulación quirúrgica se asoció con } \\
\text { una tasa significativamente reducida de } \\
\text { complicaciones, incluyendo desgarros } \\
\text { de la cápsula posterior y prolapso } \\
\text { vítreo }\end{array}$ \\
\hline $\begin{array}{l}\text { Siu- } \\
\text { Chung } \\
\text { et al } \\
2018^{21}\end{array}$ & $\begin{array}{l}\text { Estudio prospectivo, } \\
\text { transversal, multicén- } \\
\text { trico (ICO-OSCAR) }\end{array}$ & $\begin{array}{l}\text { Eyesi } \\
\text { Catarata }\end{array}$ & 22 residentes & $\begin{array}{l}\text { En los análisis multivariados, el } \\
\text { entrenamiento de simulación se asoció } \\
\text { significativamente con puntuaciones } \\
\text { de dificultad más bajas en el agrie- } \\
\text { tamiento/corte núcleos }(p=0.03) \text {, la } \\
\text { capsulorrexis }(p=0.02) \text { y la rotación/ } \\
\text { manipulación }(p=0.01)\end{array}$ & $\begin{array}{l}\text { Los residentes que habían completado } \\
\text { el entrenamiento de simulación Eyesi } \\
\text { tenían mayor confianza en la realiza- } \\
\text { ción de las tareas más difíciles percibi- } \\
\text { das durante la facoemulsificación }\end{array}$ \\
\hline
\end{tabular}


reales, encontramos algunos estudios interesantes. Belyea y sus colegas en un estudio de caso prospectivo y comparativo concluyeron que el grupo que usaba Eyesi tenía un tiempo de faco medio significativamente menor con un $\mathrm{p}=$ 0.02, un tiempo de faco ajustado y una potencia porcentual de faco menor con un $\mathrm{p}=0.001$ para ambas variables, lo que se traduce en una mejora en sus habilidades quirúrgicas y un acortamiento de la curva de aprendizaje. ${ }^{14}$

McCannel y colaboradores realizaron un estudio que buscaba evaluar el efecto de un currículum intensivo de capacitación en capsulorrexis (CITC) sobre las tasas de capsulorrexis curvilínea (CCC) errante y continua durante la cirugía de cataratas entre los cirujanos residentes. Se concluyó que el entrenamiento de simulación quirúrgica usando RV con el CITC en Eyesi redujo la tasa de capsulorrexis errante $(p=0.0001)$ en un 68\% (3.2 veces) entre el grupo de cohorte (línea de base) y el grupo de cohorte postintervención. ${ }^{15}$

Bergqvist y su equipo establecieron un programa de entrenamiento sistemático para ser incluido en la malla curricular en las residencias de oftalmología. Encontraron una mejora en la capsulorrexis ( $p<0.05)$, en la puntuación general $(p<0.01)$, y menos rupturas y daño posterior a la cápsula por ultrasonido $(p=0.05)$. También descubrieron más eficiencia con el tiempo ( $p$ $<0.01)$. Se demostró que el entrenamiento repetitivo con un programa de entrenamiento sistemático, basado en módulos validados en el simulador Eyesi, mejora las habilidades de cirugía simulada de cataratas. ${ }^{16}$ Starapoli y colegas muestran resultados similares con menores tasas

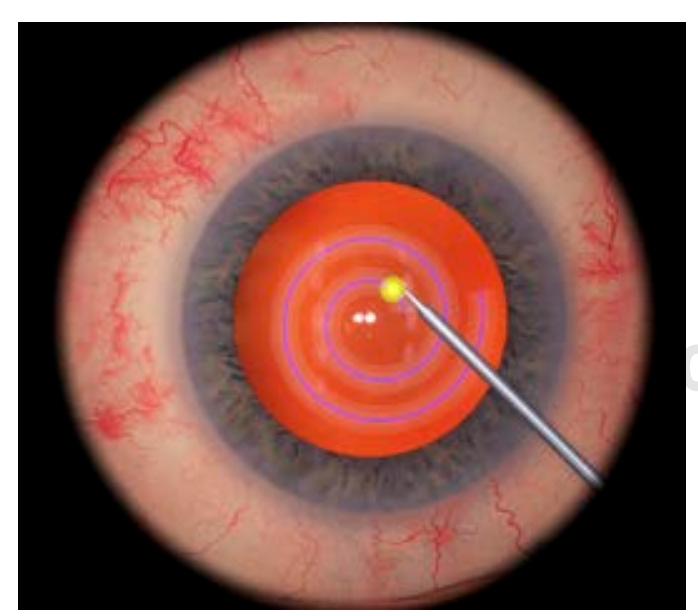

Figura 2: Módulo de antitemblor VR Eyesi.

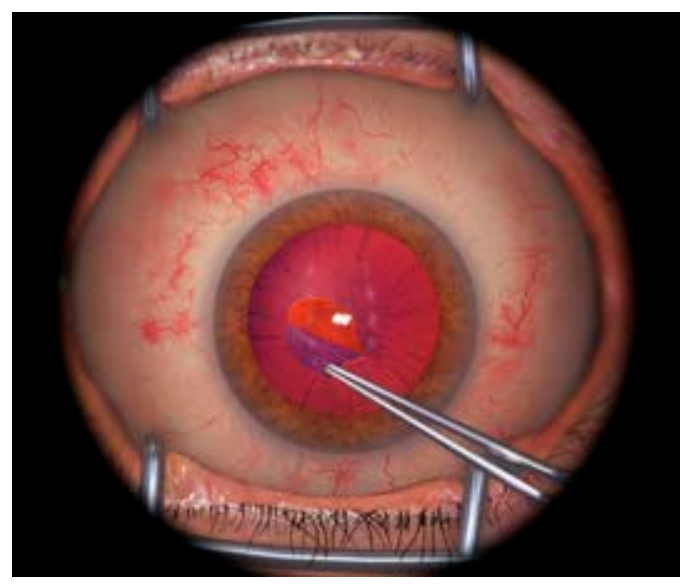

Figura 3: Módulo de capsulorrexis VR Eyesi.

de complicaciones en el grupo simulador $(\mathrm{p}=$ 0.037) y menos desgarro de la cápsula posterior y prolapso vítreo $(p=0.032){ }^{17}$

Thomsen y su grupo de investigadores realizaron un estudio en el que demostraron la validez de una prueba de rendimiento de siete módulos en el Eyesi y determinaron que la prueba era útil y confiable y podía reproducirse. ${ }^{18}$ El mismo autor en otro estudio evaluó el efecto del entrenamiento basado en el dominio de la realidad virtual sobre el rendimiento real de la cirugía de cataratas. También definió qué cirujanos se benefician del entrenamiento de realidad virtual usando Eyesi. Descubrió que tanto los cirujanos novatos como aquéllos con experiencia intermedia (menos de 75 cirugías) se beneficiaron de la capacitación al mejorar su desempeño $(p<0.05) .^{19}$

Otro aspecto muy importante al momento de la cirugía está relacionado con la confianza del cirujano. Siu-Chung y colaboradores descubrieron que aquellos residentes que se entrenaron con realidad virtual y que completaron el entrenamiento de simulación Eyesi tenían mayor confianza en la realización de las tareas más difíciles durante la facoemulsificación. ${ }^{21}$

PhacoVision es un simulador de realidad virtual para la técnica de cirugía de cataratas usando facoemulsificación. Algunos de los estudios realizados para este simulador destacan el desarrollo de índices integrados para monitorear el desempeño de los usuarios durante el entrenamiento en cirugía de cataratas. ${ }^{22,23}$ En la revisión sistemática no encontramos estudios relacionados con la validez constructiva de sus módulos.

Con respecto al MicroVisTouch, este simulador es el único dispositivo en el mercado que ha 
incorporado una interfaz de retroalimentación táctil. Se ha comprobado que la implementación de esta retroalimentación táctil proporciona una experiencia operativa más realista. ${ }^{24}$

Skilder realizó un estudio para evaluar el simulador MicroVisTouch, como una herramienta de evaluación en el rendimiento de la capsulorrexis en la cirugía de cataratas. Se evaluaron cuatro variables, circularidad, precisión, fluidez y rendimiento general. En este estudio, se encontró que los puntajes promedio en todas las métricas medidas mostraron una mejora estadísticamente significativa, con la excepción de la circularidad. Asimismo, se observó la reducción en la desviación estándar y la mejora en la capacidad del proceso en un seguimiento de seis meses. ${ }^{25}$

Al querer comparar el uso de RV y el entrenamiento usando ojos artificiales en un Wetlab, Daly y su equipo realizaron un estudio comparativo entre los residentes que realizaron capacitación preoperatoria en Wetlab usando ojos artificiales, comparándolo con los resultados intraoperatorios en residentes que realizaron capacitación con RV. Se concluyó en este estudio que no hubo diferencias significativas entre los dos grupos $(p=0.06) .{ }^{26}$

\section{Importancia del desarrollo de mallas curriculares validadas para la capacitación en cirugía de catarata usando RV}

Enseñar cirugía de cataratas es una tarea delicada y compleja que enfrentan la gran mayoría de los médicos que se dedican a enseñar y apoyar programas de residencia. Esta complejidad se explica por varios factores, por ejemplo, la dificultad de enseñar microcirugía que no permite

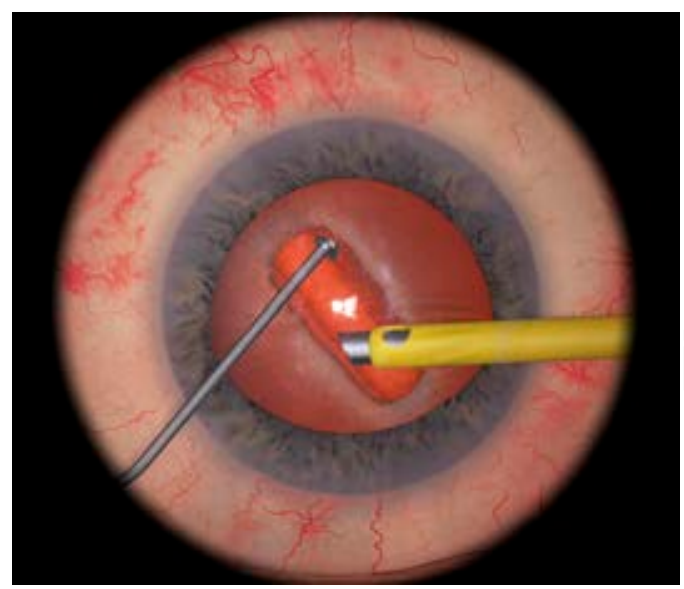

Figura 4: Módulo de cracking VR Eyesi. realizar la cirugía simultáneamente con el médico tratante; los pacientes están despiertos y alertas y finalmente las consecuencias dañinas si ocurre una complicación. ${ }^{27}$

Con respecto a este asunto, la Universidad de lowa ha publicado varios artículos sobre la importancia de la implementación de un currículum quirúrgico y las competencias que finalmente lo relacionan con los resultados quirúrgicos.

Lee y colaboradores publicaron su experiencia de cinco años con la implementación obligatoria del Consejo de Acreditación de Educación Médica para Graduados (ACGME). En este estudio implementaron 10 herramientas de evaluación de competencias básicas: 1) el plan de estudios de residencia de la Universidad de lowa, 2) observación directa utilizando el ejercicio clínico oftálmico (OCEX), 3) club de revista, 4) evaluación de múltiples fuentes, 5) una forma de evaluación global de la facultad modificada de la escala Dreyfus, 6) el currículum quirúrgico de cataratas de lowa, 7) el plan de estudios de láser oftálmico de lowa, 8) exámenes tradicionales escritos y orales, 9) ejercicios y proyectos de autorreflexión, y 10) cartera de estudiantes. De estas 10 herramientas de evaluación de competencias, evaluaron los obstáculos y buscaron formas de resolverlos. Dentro de todas estas competencias, el currículum quirúrgico de cataratas de lowa en estudios posteriores demostró ser muy útil y reproducible. ${ }^{28}$

Complementando lo anterior, Rogers y su grupo realizaron un estudio retrospectivo comparando los resultados quirúrgicos de los residentes cinco años antes de la implementación del plan de estudios de cirugía de cataratas de lowa y después de cuatro años de la implementación de este plan de estudios. Los resultados muestran una disminución en la incidencia de ruptura de la cápsula posterior y/o pérdida vítrea que varía de $7.17 \%$ (preimplementación) a 3.77\% (postimplementación) ( $p<0.001){ }^{29}$ El entrenamiento quirúrgico en Wetlab se considera uno de los componentes más importantes en la educación oftálmica, y por lo tanto, el ACGME exige el acceso a uno o un simulador quirúrgico. ${ }^{30}$ Por ejemplo, la evaluación estructurada objetiva de la habilidad quirúrgica de cataratas (OSACSS) ${ }^{31}$ y la evaluación estructurada objetiva de las habilidades quirúrgicas técnicas (OSATS) ${ }^{32}$ se han utilizado para evaluar el rendimiento quirúrgico en los residentes.

Selvander y su equipo utilizaron el uso de estas dos plataformas para evaluar el rendimiento en la realización de la capsulorrexis utilizando Eyesi. ${ }^{13}$ 
Un aspecto esencial al evaluar el proceso educativo en la simulación es el debriefing. Se han desarrollado diferentes modelos de debriefing, Eppich y Cheng desarrollaron un marco conceptual integrado para un enfoque combinado para el debriefing llamado PEARLS (Promoting Excellence And Reflective Learning in Simulation). Las estrategias educativas utilizadas durante la sesión informativa son: autoevaluación del alumno, facilitar el debate centrado y proporcionar información en forma de retroalimentación y/o enseñanza.

El marco de información de PEARLS se divide en tres fases: reacciones, descripción y análisis/ resumen..$^{20}$ Uno de los objetivos más importantes del debriefing es que los alumnos reflexionen y den sentido a su experiencia de simulación y generen un aprendizaje significativo que se traduzca en práctica clínica. Con respecto a los objetivos, el marco PEARLS y el guion de informes respaldan y promueven la coherencia dentro de los programas de simulación. ${ }^{20}$

El modelo 3D de Debriefing es un marco basado en la teoría del aprendizaje experimental y estrategias comunes de debriefing. Se compone de tres fases: desactivación, descubrimiento y profundización. Se centra en todo el proceso de aprendizaje con una importancia vital en el análisis posterior a la experiencia. Este modelo se puede utilizar para mejorar la experiencia de aprendizaje después de una capacitación real o simulada y poder analizar cada fase, entregando objetivos claros que serán desarrollados conjuntamente por el educador y el estudiante. ${ }^{33}$

Teniendo en cuenta lo mencionado anteriormente, entendiendo la importancia de la realidad virtual en el entorno de simulación, la implementación de estos modelos debe incluirse dado su impacto en el proceso de enseñanza.

\section{Análisis de costos en la simulación de facoemulsificación usando realidad virtual en programas de capacitación en oftalmología}

Lowry y colaboradores descubrieron que el número de residentes en un programa es un importante predictor de ahorro de costos. Esto mostró una amplia gama de reducciones de costos entre los programas de residencia. Por ejemplo, un pequeño programa con dos residentes en capacitación durante cinco años probablemente ahorrará $\$ 5,502$ de la mano de una mayor eficiencia operativa. Por otro lado, en un gran programa con nueve residentes a lo largo de 10 años, esto ahorraría los $\$ 46,117$ esperados. Aunque es posible ahorrar costos, es necesario realizar más estudios para evaluar la justificación en la compra o no de un simulador evaluando el impacto en los costos en la capacitación de los residentes. ${ }^{20}$

\section{DISCUSIÓN}

El aumento en la popularidad del uso de VR en el entrenamiento quirúrgico de residencia es un hecho. Esto se refleja en el aumento de la implementación de realidad virtual en los programas de residencia en oftalmología en todo el mundo. Desde la implementación de la simulación de realidad virtual como una herramienta de capacitación en los programas de oftalmología, existe una falta de evidencia sobre su efectividad y el significado real en el impacto de las habilidades quirúrgicas en la vida real.

Una de las preguntas más importantes es si el entrenamiento de RV es capaz de reducir la incidencia de complicaciones quirúrgicas en pacientes reales. Descubrimos que la plataforma más estudiada era la Eyesi. Múltiples estudios han demostrado que la mayoría de los módulos del Eyesi tienen un impacto positivo medible a través de su propia validez de constructo..$^{9-13}$ Aunque se ha medido su validez constructiva, los estudios generalmente carecen de seguimiento a largo plazo $y$, por otro lado, el modelo de estudio varía entre un centro y otro. Somos conscientes del desafío de implementar un modelo de análisis estandarizado, ya que el volumen y las capacidades quirúrgicas asociados con los factores de entrenamiento varían ampliamente entre las poblaciones.

Históricamente, el entrenamiento oftalmológico se ha basado en cirugías en pacientes reales y no en entornos seguros, como en un centro de simulación. La transición al uso de la realidad virtual es otro punto a considerar, ya que se necesita capacitación, comprensión y recursos para adquirirla y mantenerla. Otra pregunta importante basada en su implementación es si es una herramienta rentable. Con respecto a este punto, hoy nos faltan estudios que evalúen los costos asociados y su impacto a largo plazo. ${ }^{20} \mathrm{Es}$ de suma importancia cuando se quiere implementar un modelo de capacitación para tener el espacio, los recursos humanos y un plan de estudios efectivo. Es de suma importancia cuando se quiere implementar un modelo de capacitación tener un espacio con los recursos y materiales para hacerlo sostenible en el tiempo, recursos huma- 
nos, es decir, entrenadores capacitados y capaces de monitorear a los estudiantes. El debriefing es una herramienta indispensable en cualquier tipo de implementación asociada con una técnica de enseñanza. ${ }^{20,27-34}$

\section{CONCLUSIONES}

El uso de la realidad virtual en cirugías de cataratas es una herramienta que ya ha demostrado su validez de constructo. Consideramos que es una alternativa importante (complemento) al entrenamiento basado en ojos artificiales o animales.

Se necesitan estudios a más largo plazo con diseños más estandarizados para evaluar su impacto real y rentabilidad.

\section{REFERENCIAS}

1. Khalifa YM, Bogorad D, Gibson V, Peifer J, Nussbaum J. Virtual reality in ophthalmology training. Surv Ophthalmol. 2006; 51: 259-273.

2. Merril JR, Notaroberto NF, Laby DM, et al. The ophthalmic retrobulbar injection simulator (ORIS): an application of virtual reality to medical education. IEEE proceedings of annual symposium computer applications in medical care. Piscataway: IEEE Service Center; 1992. pp. 702-706.

3. Hunter IW, Tilemachos D, Lafontaine SR, et al. A tele operated microsurgical robot and associated virtual environment for eye surgery. Presence. 1993; 2: 265-280.

4. Hoznek A, Katz R, Gettman M, et al. Laparoscopic and robotic surgical training in urology. Curr Urol Rep. 2003; 4: 130-137.

5. McCloy R, Stone R. Science, medicine, and the future. Virtual reality in surgery. BMJ. 2001; 323: 912-915.

6. Letterie GS. Medical education as a science: the quality of evidence for computer-assisted instruction. Am J Obstet Gynecol. 2003; 188: 849-853.

7. Satava RM, Fried MP. A methodology for objective assessment of errors: an example using an endoscopic sinus surgery simulator. Otolaryngol Clin North Am. 2002; 35: 1289-1301.

8. Rowe R, Cohen RA. An evaluation of a virtual reality airway simulator. Anesth Analg. 2002; 95: 62-66.

9. Mahr MA, Hodge DO. Construct validity of anterior segment anti- tremor and forceps surgical simulator training modules; attending versus resident surgeon performance. J Cataract Refract Surg. 2008; 34: 980-985.

10. Selvander M, Asman P. Virtual reality cataract surgery training: learning curves and concurrent validity. Acta Ophthalmol. 2012; 90: 412-417.

11. Privett B, Greenlee E, Rogers G, Oetting TA. Construct validity of a surgical simulator as a valid model for capsulorhexis training. J Cataract Refract Surg. 2010; 36: 1835-1838.

12. Feudner EM, Engel C, Neuhann IM, Petermeier K, Bartz-Schmidt KU, Szurman P. Virtual reality training improves wet-lab performance of capsulorhexis: results of a randomized, controlled study. Graefes Arch Clin Exp Ophthalmol. 2009; 247: 955-963.

13. Selvander M, Asman P. Cataract surgeons outperform medical students in Eyesi virtual reality cataract surgery: evidence for construct validity. Acta Ophthalmol. 2013; 91: 469-474.

14. Belyea DA, Brown SE, Rajjoub LZ. Influence of surgery simulator training on ophthalmology resident phacoemulsification performance. J Cataract Refract Surg. 2011; 37: 1756-1761.

15. McCannel CA, Reed DC, Goldman DR. Ophthalmic surgery simulator training improves resident performance of capsulorhexis in the operating room. Ophthalmology. 2013; 120 (12): 2456-2461.

16. Bergqvist J, Person A, Vestergaard A, Grauslund J. Establishment of a validated training programme on the Eyesi cataract simulator. A prospective randomized study. Acta Ophthalmol. 2014; 92: 629-634.

17. Starapoli PC, Gregori NZ, Junk AK, et al. Surgical simulation training reduces intraoperative cataract surgery complications among residents. Simul Healthtc. 2018; 13 (1): 11-15.

18. Thomsen ASS, Kiilgaard JF, Kjaerbo $\mathrm{H}$, et al. Simulation-based certification for cataract surgery. Acta Ophthalmol. 2015; 93 (5): 416-421.

19. Thomsen ASS, Bach-Holm D, Kjaerbo H, et al. Operating room performance improves after proficiency-based virtual reality cataract surgery training. Ophthalmology. 2017; 124 (4): 524-531.

20. Lowry EA, Porco TC, Naseri A. Cost analysis of virtual-reality phacoemulsification simulation in ophthalmology training programs. J Cataract Refract Surg. 2013; 39 (10): 1616-1617.

21. Ng DS, Sun Z, Young Al, et al. Impact of virtual reality simulation on learning barriers of phacoemulsification perceived by residents. Clin Ophthalmol. 2018; 12: 885-893.

22. Söderberg PG, Laurell CG, Nordqvist P, Skarman E, Nordh L. Virtual cataract surgery: clinical evaluation. SPIE Proc. 200; 4951: 62-66.

23. Söderberg PG, Laurell CG, Simawi W, Skarman E, Nordqvist P, Nordh L. Performance index for virtual reality phacoemulsification surgery. SPIE Proc. 2007; 6426 (1B): 1-9.

24. Kozak I, Banerjee P, Luo J, Luciano C. Virtual reality simulator for vitreoretinal surgery using integrated OCT data. Clin Ophthalmol. 2014; 8: 669-672.

25. Sikder S, Luo J, Banerjee PP, Luciano C, et al. The use of a virtual reality surgical simulator for cataract surgical skill assessment with 6 months of intervening operating room experience. Clin Ophthalmol. 2015; 9: 141-149.

26. Daly MK, Gonzalez E, Siracuse-Lee D, et al. Efficacy of surgical simulator training versus traditional wetlab training on operating room performance of ophthalmology residents during the capsulorhexis in cataract surgery. J Cataract Refract Surg. 2013; 39: 1734-1741.

27. Ament CS, Henderson BA. Optimizing resident education in cataract surgery. Curr Opin Ophthalmol. 2011; 22 (1): 64-67.

28. Lee AG, Oetting T, Beaver HA, et al. The ACGME outcome project in ophthalmology: practical recommendations for overcoming the barriers to 
local implementation of the national mandate. Surv Ophthalmol. 2009; 54: 507-517.

29. Rogers GM, Oetting T, Lee AG, et al. Impact of a structured surgical curriculum on ophthalmic resident cataract surgery complication rates. J Cataract Refract Surg. 2009; 35: 1956-1960.

30. Accreditation Council for Graduate Medical Education. ACGME program requirements for graduate medical education in ophthalmology. 2007.

31. Saleh GM, Gauba V, Mitra A, Litwin AS, Chung AK, Benjamin L. Objective structured assessment of cataract surgical skill. Arch Ophthalmol. 2007; 125: 363-366.

32. Martin JA, Regehr G, Reznick R, MacRae H, Murnaghan J, Hutchison C, et al. Objective structured assessment of technical skill (OSATS) for surgical residents. Br J Surg. 1997; 84: 273-278.

33. Zigmont JJ, Kappus LJ, Sudikoff SN. The 3D model of debriefing: defusing, discovering, and deepening. Semin Perinatol. 2011; 35 (2): 52-58.
34. Eppich W, Cheng A. Promoting excellence and reflective learning in simulation (PEARLS): development and rationale for a blended approach to health care simulation debriefing. Simul Healthc. 2015; 10 (2): 106-115.

35. Podbielski DW, Noble J, Gill HS, Sit M, Lam WC. A comparison of hand- and foot-activated surgical tools in simulated ophthalmic surgery. Can J Ophthalmol. 2012; 47 (5): 414-417.

36. Pokroy R, Du E, Alzaga A, et al. Impact of simulator training on resident cataract surgery. Graefes Arch Clin Exp Ophthalmol. 2013; 251 (3): 777-781.

Correspondencia:

Dr. Andreas Di-Luciano R.

Orbis International

520 8th Avenue, 12th Floor

New York, NY 10018.

E-mail: andreasdilu@gmail.com 